\title{
MOTIVACIÓN, ANSIEDAD, CONFIANZA, AGRADO Y UTILIDAD. LOS FACTORES QUE EXPLICAN LA ACTITUD HACIA LAS MATEMÁTICAS EN LOS ESTUDIANTES DE ECONOMÍA
}

\author{
Carlos A. Rojas-Kramer \\ Researcher Professor at Universidad Autónoma de San Luis Potosí \\ Unidad Académica Multidisciplinaria Zona Media \\ crojask@hotmail.com \\ Milka E. Escalera-Chávez \\ Researcher Professor at Universidad Autónoma de San Luis Potosí \\ Unidad Académica Multidisciplinaria Zona Media \\ milkaech@uaslp.mx \\ Elena, Moreno-García \\ Researcher Professor at UCC Business School, Universidad Cristóbal Colón \\ Veracruz, Veracruz, México \\ E-mails: elenam@ucc.mx \\ Arturo García-Santillán* \\ Researcher Professor at UCC Business School, Universidad Cristóbal Colón \\ Veracruz, Veracruz, México \\ E-mails: agarcias@ucc.mx, arturogarciasantillan@yahoo.com.mx
}

https://doi.org/10.17060/ijodaep.2017.n1.v2.875

Fecha de Recepción: 17 Marzo 2017

Fecha de Admisión: 1 Abril 2017

\section{RESUMEN}

El objetivo del estudio se centra en determinar el nivel de actitud hacia las matemáticas por parte de los estudiantes de la Facultad de Economía de la Universidad Autónoma de San Luis Potosí (UASLP). El estudio se aborda desde el paradigma cuantitativo, no experimental, transversal y exploratorio. La muestra está conformada por estudiantes de la Licenciatura en Economía que tomaron cursos de matemáticas en el año 2105. Se utilizó el cuestionario de actitud hacia la estadística de Auzmendi (1992). El principal hallazgo empírico de la percepción de los alumnos fue que el agrado es uno de los factores que más contribuye en la actitud hacia las matemáticas, contrario a la motivación, la cual se demostró que no es un elemento clave de la actitud hacia esta disciplina.

Palabras claves: Actitud matemática, Ansiedad, Utilidad, Agrado, Motivación

Clasificación JEL: A22, C02. 


\begin{abstract}
Motivation, anxiety, confidence, pleasure and usefulness factors that really explain the attitude towards mathematics in students majoring in Economics?

The study focuses on determining the level of attitude towards mathematics by students of the Faculty of Economics at the Universidad Autónoma de San Luis Potosi (UASLP), Mexico. The study is approached from the quantitative paradigm, not experimental, transversal and exploratory. The sample consisted of college students majoring in Economics who coursed mathematics during 2105. The attitude toward statistic s test from Auzmendi (1992) was used. The main empirical finding of perception of students was that the pleasure is one of the factors that contribute to the attitude towards mathematics, contrary to motivation, which showed that it is not a key attitude to this discipline.
\end{abstract}

Keywords: Mathematics, attitude, anxiety, utility, liking, motivation.

JEL Clasification: A22, C02.

\title{
1. INTRODUCCIÓN
}

En Economía, las matemáticas desempeñan un papel importante ya que un gran porcentaje de los temas, están expresados en términos matemáticos. Particularmente, en muchas instituciones de educación superior vinculadas a la ortodoxia neoclásica, este porcentaje es muy alto. Asimismo, el avance en las tecnologías de información ha permitido incluir las técnicas de optimización y métodos estadísticos en la mayoría de los programas de la carrera de economía. Así, la enseñanza de muchos conceptos económicos a través de un lenguaje matemático se ha hecho indispensable tanto en la teoría como en la práctica económica.

En el caso mexicano de manera particular, en los estudiantes de nivel superior existe un porcentaje alto de incompetencia escolar en matemáticas. Algunos autores, como: De la Peña (2002), Velázquez (2008), Sosa (2009) y Gómez (2009), han revelado la situación actual de los estudiantes a nivel superior con relación a su rendimiento en matemáticas. Específicamente, De la Peña (2002) mostró que el $40.5 \%$ de los estudiante de la Universidad Nacional Autónoma de México (UNAM) no son competentes en el área de matemáticas. En este mismo sentido Velázquez (2008) señala que el $76 \%$ de los estudiantes de la carrera de administración de la Universidad Autónoma de Chihuahua no cuentan con habilidades para resolver problemas matemáticos. Por otra parte, en un estudio realizado por Sosa (2009), encontró que el 90\% de los estudiantes de la Facultad de Contaduría y Administración de la UASLP carecen de conocimientos básicos de matemáticas. Otro dato lo revela Gómez (2009), quien demostró que un porcentaje amplio de estudiantes de matemáticas de la Universidad Veracruzana, Campus Coatzacoalcos, presentan deficiencias considerables.

Por otro lado, González (2000) deja ver que la actitud de los estudiantes es un factor que determina la disposición para aprender, la cual influye en su comportamiento. Al respecto, Colorado, Juárez y Hernández (2011) en su estudio conciben la actitud hacia las matemáticas como un cúmulo de capacidades que las personas exteriorizan para admitir o no, determinadas capacidades. El factor actitudinal puede estar determinado por circunstancias, episodios o incidentes críticos para la configuración de un juicio, en sus palabras señalan: "que la Actitud refleja las percepciones de las personas respecto de su entorno; que resulta de gran de interés para diferentes enfoques" (sic).

En relación a la variable actitud, Auzmendi, (1992) diseñó una escala para medir la actitud del alumno hacia la estadística, en la cual propuso una serie de factores (utilidad, agrado, ansiedad, motivación, confianza), misma escala que además permite medir la actitud hacia las matemáticas en lo general. Es a partir de este modelo que se plantea la pregunta principal del estudio en los siguientes términos: ¿Existe un conjunto de variables que permitan identificar la actitud hacia las matemá- 
ticas en los estudiantes de la Facultad de Economía de la UASLP?, para dar respuesta a esta pregunta se establece como objetivo del estudio: identificar los factores que los estudiantes de economía perciben sobre la actitud hacia las matemáticas. Con lo anterior se obtendrá evidencia empírica que permita a los profesores de matemáticas, identificar áreas de mejora y de oportunidad, con el fin de generar otras estrategias didácticas en esta disciplina, todo ello, con el fin de mejorar en estos indicadores de mejora en el aprendizaje de la matemática.

Es muy importante para las instituciones educativas (públicas y privadas) contar con información de manera permanente, esto con el fin de actualizar su oferta académica y particularmente los contenidos de los planes de estudio. Además como se mencionó anteriormente esta información puede ayudar tanto a profesores como alumnos hacia un mejor proceso de enseñanza-aprendizaje de las matemáticas. De manera particular les proporcionaría habilidades y herramientas al estudiante de la carrera de economía para un mejor desempeño académico y en su vida profesional (Gil, Blanco y Guerrero, 2005, y Cardoso, Vanegas y Cerecedo, 2012). Recordemos que actualmente los organismos encargados de diseñar las políticas educativas en varios países están enfocados en la transmisión de habilidades a los jóvenes para que éstos desarrollen su potencial y puedan participar en la resolución de problemas en su ámbito laboral.

A continuación se lleva a cabo una breve revisión a la literatura especializada que aborda la medición del constructo sobre actitud de los estudiantes hacia la matemática. En un siguiente apartado, se construye el marco teórico que se emplea para la medición de la actitud hacia las matemáticas, posteriormente se presenta la metodología utilizada; los participantes, la descripción de la muestra, el instrumento aplicado y la técnica estadística de medición. Por último se presentan los resultados y conclusiones, resaltando las limitaciones de la investigación.

\section{REVISIÓN DE LA LITERATURA}

Aun cuando las matemáticas tienen gran importancia dentro de la formación de los estudiantes, ellos consideran que estos cursos son de los más difíciles de estudiar. Por lo tanto, es común observar en el estudiante que hay cierto rechazo e incluso en ocasiones, le teme a esta disciplina, lo que trae consigo que se le dificulta aprender nuevos conceptos (Aliasgar, Riahini y Mojdehavar (2010). Esta actitud negativa por parte del estudiante conduce a un estado de ansiedad, algunas veces muy alto, que obstaculiza el aprendizaje (Stubblefield, 2006 y Kargara, Tarmizi y Bayat, 2010).

Por otro lado, Fenemma y Sherman $(1976,1978)$, Sherman y Wither (2003) conceptualizan el término ansiedad como un estado de angustia que disminuye el razonamiento matemático, rendimiento y las actitudes en el estudiante, además que los lleva a evitar o no elegir cursos con contenido matemático. Entre las causas principales de la ansiedad hacia las matemáticas se cuentan la baja autoestima y el miedo al fracaso, lo cual disminuye la capacidad del estudiante de procesar la información recibida y, en consecuencia, el estudiante se obstaculiza a sí mismo para resolver problemas relacionados con las matemáticas.

Otros estudios han revelado una relación significativa entre el razonamiento matemático, la ansiedad matemática y las actitudes matemáticas. Han mostrado que los estudiantes con una actitud positiva hacia esta disciplina, están más motivados y comprometidos en sus clases y entienden de manera más sencilla el material de sus clases, a diferencia de los estudiantes que mantienen una actitud negativa (Garry, 2005).

El desempeño en las habilidades matemáticas, no sólo se concentran en el conocimiento, sino también en la habilidad procedimental y la dimensión actitudinal y metacognitiva, además hay que contemplar tanto la dimensión social como la psicológica que poseen las competencias matemáticas y su correspondiente enseñanza. 
En el cuadro 1 se muestran algunos estudios que se han realizado en torno al tema y los distintos enfoques que se le han dado.

Cuadro 1.

Estudios empíricos de actitud hacia las matemáticas

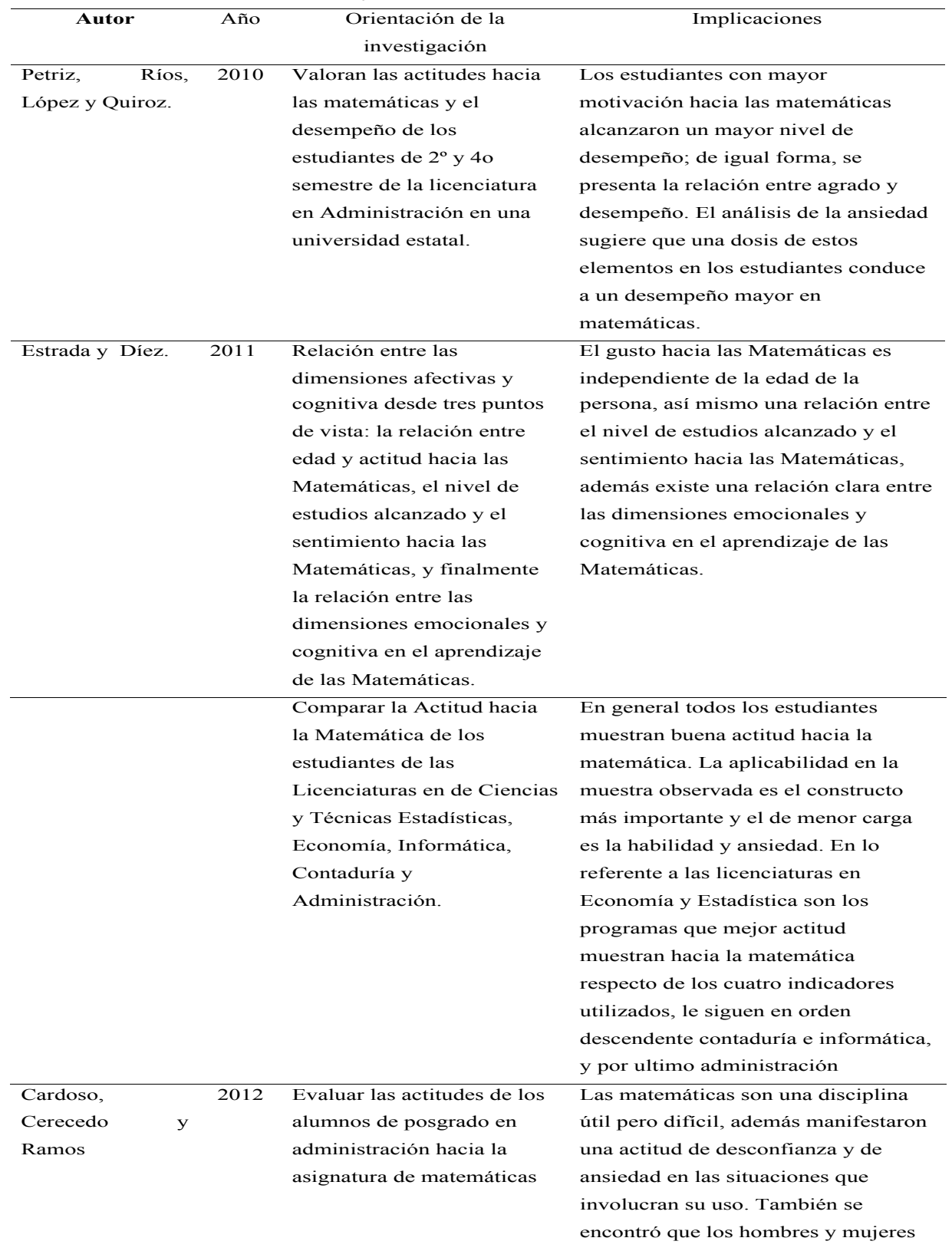




\begin{tabular}{|c|c|c|}
\hline & & $\begin{array}{l}\text { que inician el posgrado en } \\
\text { administración difieren en su nivel de } \\
\text { ansiedad al realizar actividades } \\
\text { relacionadas con las matemáticas. }\end{array}$ \\
\hline $\begin{array}{l}\text { Dörfer, Duque y } 2016 \\
\text { Soledad. }\end{array}$ & $\begin{array}{l}\text { Aplican y validan la } \\
\text { fiabilidad de la escala de } \\
\text { actitudes hacia las } \\
\text { matemáticas EAM, } \\
\text { elaborada por Elena } \\
\text { Auzmendi (1992) }\end{array}$ & $\begin{array}{l}\text { Afirman que la escala concibe la } \\
\text { actitud hacia las matemáticas } \\
\text { mediante las subescalas de la } \\
\text { ansiedad, el agrado, la utilidad, la } \\
\text { motivación y la confianza. }\end{array}$ \\
\hline
\end{tabular}

Fuente: elaborado con fuentes consultadas

Como se puede observar en el Cuadro 1, algunos estudios teóricos disponibles en la literatura especializada, abordan distintos enfoques que buscan explicar la relación de las matemáticas con diferentes factores que afectan el rendimiento de los estudiantes en ésta disciplina. Es por ello que para este estudio, se sigue el trabajo de Auzmendi (1992) con respecto a la actitud hacia la matemática.

En este sentido, en relación al constructo de ansiedad Fennema y Sherman $(1976,1978)$ dejan ver que la ansiedad matemática se desarrolla con "un sentimiento de ansiedad, terror, nerviosismo y síntomas físicos asociados que surgen al hacer matemáticas". En lo reportado en sus investigaciones empíricas señalan que los estudiantes que experimentan menor grado de ansiedad ante las matemáticas son los que también tenían una actitud más favorable ante ellas. Por último, estos autores indican que no es suficiente la disposición que un alumno tenga para el aprendizaje matemático, sino también hay que tomar en cuenta sus habilidades para perseguir procedimientos lógicos.

En este mismo sentido, Cardoso, Vanegas y Cerecedo, (2012) mencionan que la ansiedad hacia las matemáticas es la conducta de un estudiante que se manifiesta por un miedo excesivo a cometer errores, disminuyendo el grado de atención y por ende a un razonamiento deficiente; la motivación es el comportamiento de una persona dirigido hacia una actividad preferencial.

En lo que se refiere al constructo agrado por las matemáticas, éste se refiere al grado que un estudiante se siente feliz con relación al estudio de las matemáticas (Santrock, 2010). La confianza hace referencia a la seguridad o esperanza firme que una persona tiene de un objeto 0 de sí misma ante una determinada situación, y, por último, la utilidad es el valor que un individuo da a un objeto (el valor que un alumno da a las matemáticas).

\section{FUNDAMENTO TEÓRICO}

Para examinar la actitud hacia las matemáticas en esta investigación se toma como base la teoría de Weiner (1985), la cual es útil para explicar la conducta en diversas situaciones. Esta teoría explica que frente a un acontecimiento se produce una reacción general positiva o negativa, según se perciba éxito o fracaso y se crean, posteriormente, una serie de emociones diferentes (orgullo, desesperanza, culpabilidad, ira, autoestima, ansiedad, confianza, motivación), directa 0 indirectas que condiciona la conducta del sujeto. La combinación de dichos criterios determina la percepción en el estudiante y que a su vez influenciará la conducta (Beltran y Avaraez. 1995)

Además, esta investigación se sustenta en el modelo propuesto por Auzmendi (1992) que permite evaluar las emociones que Wiener (1985) señala en su teoría. Auzmendi (1992) propone una estructura de 5 constructos que permiten medir la actitud hacia la estadística, un constructo para el 
componente cognitiva concerniente con la utilidad y cuatro para los elementos que miden la parte afectiva (ansiedad, confianza, agrado y motivación), en el cuadro 2 se presenta la descripción de las dimensiones y en la figura 1 el modelo.

Cuadro 2

Descripción y codificación de los constructos

\begin{tabular}{|l|l|c|}
\hline \multicolumn{1}{|c|}{ Constructo } & \multicolumn{1}{|c|}{ Conceptualización } & Codificación \\
\hline Motivación & $\begin{array}{l}\text { Es lo que siente el estudiante hacia el estudio y utilización de } \\
\text { la matemática }\end{array}$ & $X_{1}$ \\
\hline Utilidad & $\begin{array}{l}\text { Es el valor que el estudiante otorga a las matemáticas para su } \\
\text { futura vida profesional. }\end{array}$ & $X_{2}$ \\
\hline Ansiedad & $\begin{array}{l}\text { Se refiere al temor que el estudiante manifiesta ante la } \\
\text { asignatura de matemáticas. }\end{array}$ & $X_{3}$ \\
\hline Confianza & $\begin{array}{l}\text { Es el sentimiento de confianza que provoca la habilidad en la } \\
\text { matemática. }\end{array}$ & $X_{4}$ \\
\hline Agrado & Hace referencia al disfrute que provoca el trabajo matemático & $X_{5}$ \\
\hline
\end{tabular}

Fuente: elaborado con datos de Auzmendi (1992)

Figura 1

Modelo propuesto por Auzmendi (1992)

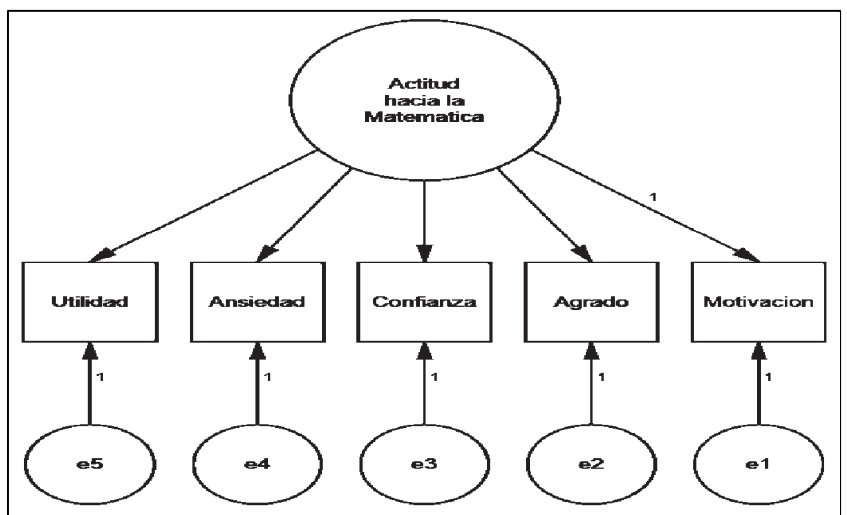

\section{METODOLOGÍA}

El estudio es no experimental transversal y factorial exploratorio ya que se pretende analizar la interrelación entre un grupo de variables y explicarlas en términos de sus dimensiones (Hair, 1999). Es transversal teniendo en cuenta que la obtención de datos se da sólo una vez durante un tiempo determinado. Para fines de esta investigación seguimos el procedimiento utilizado por EscaleraChávez, García-Santillán, y Venegas-Martínez (2014), de ahí que la muestra es no probabilística por- 
que la elección de los elementos no depende de la probabilidad, sino de causas relacionadas con las características de la investigación. Los criterios de selección de la muestra fueron incluir a estudiantes que hayan completado al menos un curso de matemáticas en el programa de licenciatura en economía en la UASLP. Además se buscó que estuvieran disponibles en la institución al momento de aplicar la encuesta y sobre todo que estuvieran de acuerdo en responderla.

De esta forma, la muestra de estudio se conformó de 75 estudiantes de la carrera de economía en el primer y cuarto semestre del ciclo escolar 2015-2016, y su representatividad en relación al género fue de $43.1 \%$ masculino y $56.9 \%$ femenino, cuyo rango de edad oscila entre los 17 a 25 años (cuadro 3).

Cuadro 3.

Perfil de los encuestados (año y género)

\begin{tabular}{cccc}
\hline Edad & \multicolumn{2}{c}{ Género (\%) } & Total \\
Años & Masculino & Femenino & $(\%)$ \\
\hline 17 & & 1.4 & 1.4 \\
18 & 8.3 & 8.3 & 16.7 \\
19 & 12.5 & 19.4 & 31.9 \\
20 & 5.6 & 6.9 & 12.5 \\
21 & 6.9 & 8.3 & 15.3 \\
22 & 2.8 & 11.1 & 13.9 \\
23 & 4.2 & 1.4 & 5.6 \\
24 & 1.4 & & 1.4 \\
25 & 1.4 & & 1.4 \\
\hline Total & 43.1 & 56.9 & 100.0 \\
\hline
\end{tabular}

Fuente: propia

Para el trabajo de campo se utilizó el cuestionario Auzmendi (1992) que consta de cinco factores: motivación (ítems 5, 10, 15, 20 y 25), ansiedad (ítems 2, 7, 12, 17 y 22), agrado (ítems 4, 9, 14, 19 y 24), confianza (ítems (3, 8, 13, 18 y 23) y utilidad (ítems 1, 6, 11, 20 y 21). El rango de escala se mide de 1 (bajo) a 5 (muy alto). Para el procesamiento de los datos se utilizó el programa SPSS v 22.

Para validar el instrumento se lleva a cabo el cálculo del coeficiente alfa de Cronbach ( ), el cual Hair, Anderson, Tatham y Black (1999) señalan que los coeficientes de fiabilidad tienen valores que van de 0 a 1 y cuanto más cercano esté de la unidad, es sinónimo de más alta fiabilidad. Además el alfa de Cronbach puede ser interpretado como una función del número de ítems y el promedio de las correlaciones entre los ítems, a partir de la siguiente expresión:

$$
\alpha=N * \bar{r} / 1+(N-1) * \bar{r}
$$

Dónde: $\mathrm{N}=$ Numero de ítems (o variables latentes), $\bar{r}=$ promedio de las correlaciones entre los ítems. 
Los resultados de la validación se muestran en el cuadro 4.

Cuadro 4.

Escala de Fiabilidad

\begin{tabular}{lcc}
\hline Constructo & Alfa de Cronbach & Estadísticos de fiabilidad \\
\hline Motivación & .786 & \\
Utilidad & .769 & $\mathrm{~N}$ de elementos \\
Ansiedad & .781 & 5 \\
Confianza & .745 & Alfa de Cronbach \\
Agrado & .716 & 0.799 \\
\hline
\end{tabular}

a. eliminación por lista sobre todas las variables en el procedimiento

Fuente: propia

Los resultados obtenidos por cada dimensión del constructo de actitud y el global estandarizado superan el umbral de 0.5 Por lo tanto ambos muestran un coeficiente muy aceptable de acuerdo a lo que establece Hair, et al. (1999) > 0.7 de ahí que podemos decir que el instrumento reúne las características de consistencia interna requerida para este tipo de estudios, en consecuencia la validez del test se confirma.

\section{ANÁLISIS ESTADÍSTICO Y RESULTADOS EMPÍRICOS}

En primer lugar se calculó para este grupo de variables (cuadro 2), la matriz de correlación para determinar si el análisis factorial es una técnica viable de aplicar. En el cuadro 5 se observa que de las 5 variables, 4 son significativas (al 90\%) lo que indica que es una base adecuada para realizar los siguientes análisis empíricos.

Cuadro 5.

Matriz de correlaciones

\begin{tabular}{cccccc}
\hline Variables & $X_{1}$ & $X_{2}$ & $X_{3}$ & $X_{4}$ & $X_{5}$ \\
\hline$X_{1}$ & 1 & $0.452^{* *}$ & $0.450^{* *}$ & $0.267^{*}$ & $0.378^{* *}$ \\
$X_{2}$ & & 1 & $0.167^{*}$ & $0.485^{* *}$ & $0.588^{* *}$ \\
$X_{3}$ & & & 1 & $0.521^{* *}$ & $0.489^{* *}$ \\
$X_{4}$ & & & & 1 & $0.629^{* *}$ \\
$X_{5}$ & & & & & 1
\end{tabular}

* no significativa

Posteriormente se contrasto la matriz de correlaciones con la prueba del Test de esfericidad de Bartlett, los valores de $X^{2} 10 \mathrm{gl}(130.056)$ y su significancia $($ sig. $=0.0)$ demuestran que es factible realizar ésta técnica. También el valor KMO (0.647) fue significativo ya que excede el umbral teórico sugerido mayor de 0.50 y los valores de la Medida de Suficiencia de Muestreo (MSA) para cada variable $\left(X_{1}=0.603 ; X_{2}=0.576 ; X_{3}=0.558 ; X_{4}=0.718\right.$ y $\left.X_{5}=0.759\right)$ son superiores de 0.5 . 
Posteriormente se realiza el análisis de componentes principales para su extracción, mismo análisis que deja ver que las 5 dimensiones del constructo de Actitud hacia la matemática se inscriben en un solo componente con un autovalor mayor que 1 (cuadro 6), el cual de acuerdo a las consideraciones teóricas de acuerdo al criterio de raíz latente (Hair et al, 1999), lo cual indica que es un componente apropiado para explicar los factores que contribuyen a la actitud de los estudiantes hacia las matemáticas y que explica el $55.71 \%$ de la varianza total. En apoyo a estos argumentos, se puede ver en la figura 2 en donde se presenta el gráfico de sedimentación y se observa en el contraste de caída que hay solamente un factor apropiado para explicar el escenario estudiado, que como se mencionó, está integrado por los cinco constructos (dimensiones de la actitud hacia la matemática): agrado, confianza, utilidad, ansiedad y motivación.

Figura 2.

Análisis de sedimentación

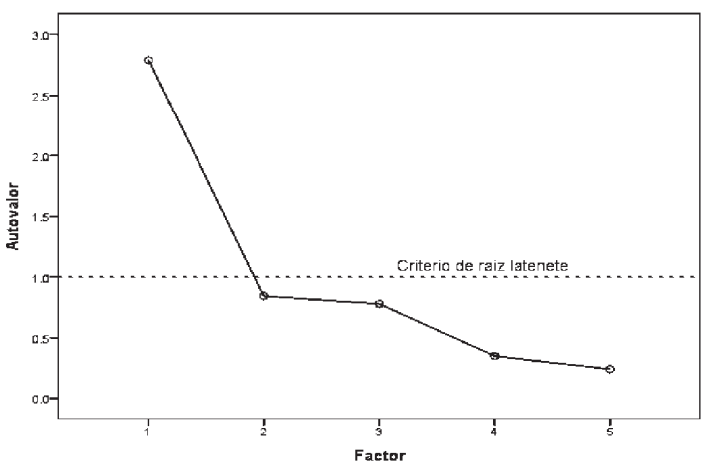

\subsection{Análisis de componentes}

En el cuadro 6 se muestra la información relativa a las cargas factoriales de cada una de las cinco dimensiones del constructo de la Actitud. En el mismo cuadro se presenta la proporción de la varianza representada por la Comunalidad ( ) resultante del cuadrado de los pesos factoriales de cada dimensión y su poder explicativo (eigenvalue) que representa el porcentaje total de la varianza explicada. Como dato importante a destacar de la información mostrada en el cuadro 6, la carga factorial del constructo agrado, es el que muestra mayor valor y la motivación es la que menos carga factorial muestra.

Cuadro 6.

Cargas factoriales, autovalor y varianza

\begin{tabular}{lc|cc}
\hline \multicolumn{1}{c|}{ Variables } & Componente & Comunalidades \\
\hline Agrado & .847 & .717 \\
Confianza & .795 & .632 \\
Utilidad & .725 & .525 \\
Ansiedad & .696 & .484 \\
Motivación & .656 & .430 \\
\hline \multicolumn{2}{c}{ Autovalor } & \multicolumn{2}{c}{2.789} \\
\hline \multicolumn{2}{c}{ Varianza total (\%) } \\
\hline
\end{tabular}

Fuente: propia 


\section{MOTIVACIÓN, ANSIEDAD, CONFIANZA, AGRADO Y UTILIDAD. LOS FACTORES QUE EXPLICAN LA ACTITUD HACIA LAS MATEMÁTICAS EN LOS ESTUDIANTES DE ECONOMÍA}

\section{CONCLUSIONES}

Al final del estudio, se logra tener evidencia concreta sobre la población que fue objeto de análisis, y podemos decir que existen cinco características visibles que los estudiantes de la facultad de economía perciben de la actitud hacia la Matemática, las cuales se agrupan en un solo factor denominado actitud. Todos los constructos tienen signo positivo, lo que sugiere que estas percepciones son muy similares en las respuestas, no actuando en sentido opuesto, lo que nos lleva a pensar en la coherencia de esta actitud, es decir, que se le da un valor importante a cada una de estas dimensiones.

De igual forma el resultado nos lleva a pensar, que la actitud hacia las matemáticas esta proporcionada por el gusto 0 el agrado que el alumno tenga hacia la disciplina, la confianza que tenga en su habilidad para las matemáticas, la utilidad que el estudiante vea en esta disciplina en su futura vida profesional, además del sentimiento de miedo que el estudiante manifiesta ante la asignaturas y de las razones que impulsan al estudiante a aprender matemáticas. Los estudiantes consideran estos factores útiles para explicar su actitud. Al respecto de la ansiedad, en un estudio GarcíaSantillán, Escalera-Chávez, Moreno-García y Santana-Villegas, (2016) demostraron que la ansiedad es un factor que impide a los estudiantes de alcanzar un buen rendimiento en el proceso de aprendizaje.

Del mismo modo, recientemente en otro estudio llevado a cabo a estudiantes de una escuela náutica en el Puerto de Veracruz, García-Santillán, Moreno-García, Schnell, and Ramos-Hernández, (2016) demostraron que la ansiedad matemática hacia las situaciones de la vida cotidiana no genera tanta ansiedad en el estudiante, no así la ansiedad ante la comprensión de problemas matemáticos y la ansiedad frente a los números de operaciones matemáticas, los cuales fueron los componentes que mayor saturación mostraron en su estudio ( 0.910 y 0.902 respectivamente) y los componentes restantes, ansiedad hacia la evaluación también obtuvieron altos porcentajes $(80 \%$ y $72.7 \%$ ), lo que implica que la mayoría de las dimensiones inciden ampliamente en la ansiedad de los alumnos hacia las matemáticas.

Similar el resultado obtenido en el estudio de García-Santillán, Edwards Wurzinger, y TejedaPeña (2015), todo esto nos lleva a pensar que este fenómeno de la ansiedad se da con más frecuencia en contextos académicos, más allá de enfrentarse a situaciones de la vida cotidiana en donde se requiere conocimiento matemático, su ansiedad disminuye notablemente, lo cual es un elemento interesante debido a que como señalan Furner y Berman (2003) los estudiantes deben darse cuenta que las matemáticas tienen aplicaciones fuera del salón de clases y es necesaria en la vida cotidiana.

Partiendo de estos referentes empíricos citados previamente y observando el resultado de este estudio en el cuadro 6, podemos inferir que si bien la ansiedad fue un factor que mostró un menor peso factorial (.696) con respecto al resto, también es cierto que está presente significativamente en el constructo de la actitud del alumno hacia la matemática, y que cuando cargan positivamente el resto de los factores del componente extraído (agrado, utilidad, motivación, confianza) esta variable sigue la misma tendencia positiva.

Este dato es importante debido a que podría auxiliar en la percepción hacia la materia y por consiguiente reducir su nivel de ansiedad al verla como una parte necesaria de su desarrollo humano en la sociedad y no únicamente como una exigencia en el contexto escolar. Si consideramos a la ansiedad como un elemento importante en el constructo de la actitud, y como ésta ha sido estudiada por diversos autores por separado, ya que se ha comprobado que niveles altos de ansiedad, son detonantes del incremento en los otros elementos del constructo de la actitud y de la propia Ansiedad (Tobias \& Weissbrod, 1980; Venkatesh-Kumar \& Karimi, 2010; Tezer \& Bozkurt, 2015; 
García-Santillán, Edwards-Wurzinger \& Tejada-Peña 2015; García-Santillán, Escalera-Chávez, Moreno-García, \& Santana-Villegas, 2015; García-Santillán, Escalera-Chávez, Moreno-García y Santana-Villegas, 2016; García-Santillán, Moreno-García, Schnell, Jutta and Ramos, 2016).

Finalmente podemos decir que ésta investigación aporta evidencia para mejorar el proceso enseñanza aprendizaje y buscar que el estudiante adquiera un aprendizaje significativo porque los estudiantes seguirán utilizando matemáticas durante su formación académica y además es importante resaltar que al ingresar al entorno laboral requiere de esta disciplina para un buen desempeño teórico y práctico de la economía. Y algo importante a destacar es lo que señala Tezer \& Bozkurt (2015) que cuando una persona tiene una actitud positiva hacia las matemáticas, que le agrade la materia, que su proceso de aprendizaje se desarrolle con agrado y no se perciba como una obligación, se creará una atmósfera placentera en el aula.

También los resultados de esta investigación permiten confirmar el objetivo planteado en esta investigación. Es decir, identificar los factores que los estudiantes de la Facultad de Economía de la Universidad Autónoma de San Luis Potosí perciben sobre la actitud hacia las matemáticas. Con base en el criterio de varianza explicada, los factores que la explican comprenden un $56 \%$ de la varianza extraída, el otro $44 \%$ está explicado por otras variables que no han sido consideradas en este modelo. Los hallazgos empíricos de este estudio contribuyen al enriquecimiento del modelo teórico propuesto por Auzmendi (1992).

Como limitante del estudio podemos señalar que la muestra fue una de las limitantes, ya que es conveniente realizar estudios con muestras mayores para precisar cuál de los factores es el más recurrente de los cinco constructos: ansiedad, agrado, motivación, utilidad o confianza. Asimismo, el trabajo puede extenderse a otros contextos y que permita con ello poder comparar varias instituciones que tengan la licenciatura en economía

\section{REFERENCIAS}

Auzmendi, E. (1992). Las actitudes hacia la matemática-estadística en las enseñanzas media y universitaria, Bilbao: Mensajero.

Beltran, J. Alvarez, JA. (1995). Piscología e la Educación. Ed Marcomo. Barcelona.

Cardoso, E., Vanegas, E. y Cerecedo, M. (2012). Diagnóstico sobre las actitudes hacia las Matemáticas del estudiantado que inicia sus estudios en tres posgrados en Administración de Empresas. Revista Electrónica Educare, 16, (2), 237-253.

Colorado. J., Juárez, S. y Hernández, ML. (2011). Actitud hacia la matemática en estudiantes del área económica administrativa. Documentos del XV Congreso Internacional de Investigación en Ciencias Administrativas, mayo. Veracruz, México.

De la Peña, J. (2002). Algunos problemas de la educación en matemáticas en México, México: Siglo XXI Drexel University.

Dörfer, C., Duque, U. \& Soledad, G. (2016). Medición de la actitud hacia las matemáticas en estudiantes de licenciatura en administración: un estudio piloto. VinculaTégica .EFA, 2(1, 1329-1348.

Escalera-Chávez, M.A., García-Santillán, A. y Venegas-Martínez, F. (2014). Modeling attitude toward Statistic with structuralequation (2014). Eurasia Journal of Mathematics, Science y Technology Education. Vol. 10 Issue (1) pp 23-31 ISSN:1305-8223, 2014.

Estrada, A y Diez J. (2011). Las actitudes hacia las Matemáticas. Análisis descriptivo de un estudio de caso exploratorio centrado en la Educación Matemática de familiares. Revista de Investigación en Educación, 9 (2), pp. 116-132.

Fennema, E y Sherman, J. (1976) Mathematics Attitudes Scales: Instruments Designed to Measure Attitudes toward the Learning of Mathematics by Females y Males Journal for Research in Mathematics Education, Vol. 7(5), 324-326. 
Fennema, E. y Sherman, J. (1978). Sex-related differences in mathematics achievement and related factors: A further study, Journal for Research in Mathematics Education. 9, 189-203, 1978.

Furner, J., \& Berman, B. (2003). Math anxiety: Overcoming a major obstacle to the improvement of student math performance. Childhood education, 170-175.

García-Santillán, A., Edwards Wurzinger, A. y Tejeda-Peña, E. (2015). What factors explain the anxiety level towards the study of mathematics among elementary school students? Mediterranean journal of Social Sciences MCSER publishing, 6(4), 564-572.

García-Santillán, A., Escalera-Chávez, M., Moreno-García, E. y Santana-Villegas, J. (2016). Factors that explains student's anxiety towards mathematics. Eurasia journal of Mathematics, Science y Technology Education, 12(2), 361-372.

García-Santillán, A., Moreno-García, E., Schnell, Jutta and Ramos, Jesica., (2016). Anxiety towards mathematics on undergraduates in a Nautical School (An empirical study in Port Veracruz) Matemáthics Education Vol. 11 (7) pp. 2418-2429

Garry, V. S. (2005). The effect of mathematics anxiety the course and career choice of high school vocational-technical education students. PhD Thesis Philadelphia. Recuperado de http://medind.nic.in/jak/t10/i1/jakt10i1p147.pdf.

Gil, N., Guerrero, E. y Blanco, L.J. (2006 b).El dominio afectivo en el aprendizaje de las Matemáticas. Revista de Investigación psicoeducativa, 27- 4

Gómez, J. (2009). Conocimientos previos de los alumnos de nuevo ingreso a la Facultad Contaduría y Administración campus Coatzacoalcos", documentos del XII Congreso Internacional sobre Innovaciones en Docencia e Investigación en Ciencias Económico Administrativas, octubre, Querétaro, México.

González, F. E. (2000). Agenda latinoamericana de investigación en Educación Matemática para el siglo XXI. Educación Matemática, 12 (1), 107-128.

Hair, J. F., Anderson, R. E., Tatham, R. L. y Black, W. C. (1999). Multivariate data analysis, fifth edition. Spain Prentice Hall.

Kargara, M., Ahmad. R. y Bayatc, S. (2010). Relationship between Mathematical Thinking, Mathematics Anxiety and Mathematics Attitudes among University Students. Procedia Social and Behavioral Sciences, 8, 537-542.

Petriz, A., Ríos, B, López C. Rosa María, \& Quiroz J. (2010). Niveles de desempeño y actitudes hacia las matemáticas en estudiantes de la Licenciatura en Administración en una universidad estatal mexicana. Revista mexicana de investigación educativa, 15(47), 1223-1249. Recuperado en 26 de septiembre de 2016, de http://www.scielo.org.mx/scielo.php?script=sci_arttext\&pid=S1405$66662010000400012 \& \operatorname{lng}=e s \& \operatorname{lng}=e s$

Tezer, M., \& Bozkurt, A. (2015). Determining Attitudes and Anxiety Levels of Students in Need of Protection Towards Mathematics Course. Procedia - Social and Behavioral Sciences (186), 269 $-273$.

Tobias, S., \& Weissbrod, C. (1980). Anxiety and mathematics: an update. Harvard Educational Review, 50(1), 63-70

Sherman, B. y Wither, D. (2003). Mathematics Anxiety and Mathematics Achievement. Mathematics Education Research Journal, 15, (2), 138-150.

Sosa, E. (2009). La resolución de problemas y el uso de software en la enseñanza de las matemáticas", documentos del XII Congreso Internacional sobre Innovaciones en Docencia e Investigación en Ciencias Económico Administrativas, octubre, Querétaro, México.

Stubblefield, L. (2006). Mathematics anxiety among GED recipients in four-year institutions. Journal of Mathematics Science \& Mathematics Education, 1, 19-23. 
Velázquez, F. (2008). Opinión de los alumnos que asistieron al curso nivelatorio de matemáticas y su resultado en la materia de matemáticas básicas en la Facultad de Contaduría y Administración de la Universidad Autónoma Chihuahua, documentos del XI Congreso Internacional sobre Innovaciones en Docencia e Investigación en Ciencias Económico Administrativas, septiembre, Guanajuato, México.

Venkatesh Kumar, G., \& Karimi, A. (2010). Mathematics Anxiety, Mathematics Performance and Overall Academic Performance in High School Students. Journal of the Indian Academy of Applied Psychology, 36(1), 147-150.

Weiner, B. (1985). An Attributional Theory of Achievement Motivation and Emotion. Psychological Review, 92(4), 548-573. 
